\title{
Effect of electron-hole asymmetry on cross-polarized excitons in carbon nanotubes
}

\author{
Seiji Uryu ${ }^{1}$ and Tsuneya Ando $^{2}$ \\ ${ }^{1}$ Department of Materials Science and Engineering, Iwate University, 4-3-5 Ueda, Morioka, Iwate 020-8551, Japan \\ ${ }^{2}$ Department of Physics, Tokyo Institute of Technology, 2-12-1 Ookayama, Meguro-ku, Tokyo 152-8551, Japan
}

(Received 26 September 2010; published 11 February 2011)

\begin{abstract}
Excitonic absorption of semiconducting carbon nanotubes for polarization perpendicular to the tube axis is theoretically studied in an effective-mass approximation including higher-order terms describing trigonal warping, curvature, lattice distortion, and electron-hole asymmetry. Asymmetry between electrons and holes causes brightening of dark excitons leading to additional low-energy peaks in optical absorption spectra. In comparison with parallel polarization, chiral-angle dependence of excitation energy is weak because of the cancellation of higher-order corrections between relevant valence and conduction bands.
\end{abstract}

DOI: 10.1103/PhysRevB.83.085404

PACS number(s): 78.67.Ch, 73.22.Lp

\section{INTRODUCTION}

The atomic structure of a carbon nanotube is uniquely specified by a chiral vector that defines the circumference of the nanotube on its development map. Excitation energies of various nanotubes approximately show linear dependence on the inverse of the circumference. Additional dependence on the direction of the chiral vector exhibits the so-called family pattern. It plays an important role in the assignment of nanotube structure ${ }^{1}$ and has been intensively studied mainly in optical properties for parallel polarization. ${ }^{1-7}$ In this paper, we theoretically study cross-polarized-light absorption of carbon nanotubes in an effective-mass approximation including higher-order corrections that can describe the family pattern.

In optical properties of semiconducting carbon nanotubes, exciton effects are prominent because of one dimension. This was first theoretically predicted for parallel polarization, ${ }^{8,9}$ in which the electric field is parallel to the axis, and later confirmed experimentally ${ }^{1,10-15}$ and theoretically. ${ }^{14-22}$ For parallel polarization, the binding energy of excitons is comparable to the band gap and a large amount of absorption intensity is focused on them.

For cross polarization, in which the field is perpendicular to the axis, the absorption is strongly affected by the depolarization effect, ${ }^{23,24}$ but the strong exciton effect causes the appearance of sharp peaks although their intensity is reduced. ${ }^{18,19,21,25,26}$ These peaks were actually observed in experiments $^{27-29}$ together with a family pattern weaker than that for parallel polarization. ${ }^{28}$

Various electronic properties of nanotubes have been theoretically clarified within an effective-mass approximation. ${ }^{7,22,30-34}$ In this scheme, higher-order corrections describe trigonal warping of energy bands, ${ }^{7,30,35}$ curvature, ${ }^{32,36}$ lattice distortion, ${ }^{32,37-40}$ and electron-hole asymmetry. ${ }^{7,41} \mathrm{~A}$ recent study on optical properties for parallel polarization revealed that these corrections can well reproduce experimental family patterns. ${ }^{7}$

Higher-order terms play different roles for perpendicular polarization. Within the lowest-order effective-mass scheme, the conduction and valence bands are symmetric with each other and cross-polarized excitons are degenerate between the $K$ and $K^{\prime}$ points at the corner of the Brillouin zone. Only the symmetric combination of these excitons becomes optically active (bright exciton), although it is strongly affected by depolarization effects and the antisymmetric combination remains optically inactive (dark exciton). ${ }^{25}$ When the degeneracy is lifted, the dark exciton becomes optically active, as has been demonstrated by the Aharonov-Bohm magnetic flux passing through the tube cross section. ${ }^{42}$

The degeneracy is actually lifted by electron-hole asymmetry present in the band structure and, therefore, such effects were already included in various calculations based on first principles and a tight-binding model. ${ }^{18,19,21,26}$ Quite recently, experimental observation of brightened dark excitons in the perpendicular polarization was reported ${ }^{43}$ and some attempts of analyzing the experiments based on an effective-mass scheme were made, ${ }^{41,43}$ although they were unsatisfactory, as will be discussed in the following.

The purpose of this paper is to systematically study the effects of various higher-order terms on cross-polarized excitons in semiconducting carbon nanotubes in the effectivemass approximation. The paper is organized as follows: The model and method are described in Sec. II. Numerical results are presented in Sec. III and discussed in Sec. IV. A summary and conclusion are given in Sec. V.

\section{MODEL AND METHOD}

\section{A. Effective-mass approximation}

In a two-dimensional graphite sheet called graphene [as shown in Fig. 1(a)], the conduction and valence bands consisting of $\pi$ states cross at $K$ and $K^{\prime}$ points [as shown in Fig. 1(b)] and electrons around these valleys are described by a $\mathbf{k} \cdot \mathbf{p}$ equation. For each valley, it is given by ${ }^{30,44,45}$

$$
\mathcal{H} \mathbf{F}(\mathbf{r})=\varepsilon \mathbf{F}(\mathbf{r}),
$$

where $\mathcal{H}$ is a $2 \times 2$ Hamiltonian matrix corresponding to $A$ and $B$ sublattices, $\mathbf{F}(\mathbf{r})$ is a two-component envelope function, $\mathbf{r}=$ $(x, y)$, the $x$ and $y$ coordinates are chosen in the circumference and the axis directions, respectively, as shown in Fig. 1(c), and $\varepsilon$ is the eigenenergy.

For the $K$ point, the Hamiltonian matrix with the energy origin at the Fermi energy is given by $7,30,35,44,45$

$$
\mathcal{H}_{K}=\left(\begin{array}{lc}
\sqrt{3} a \gamma S \hat{\mathbf{k}}^{2} / 2 & h_{K}(\hat{\mathbf{k}}) \\
h_{K}(\hat{\mathbf{k}})^{\dagger} & \sqrt{3} a \gamma S \hat{\mathbf{k}}^{2} / 2
\end{array}\right),
$$




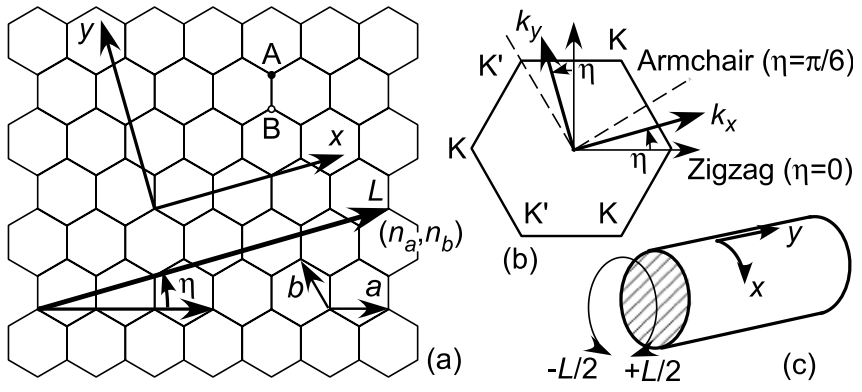

FIG. 1. Schematic illustration of (a) the lattice structure of a twodimensional graphite sheet, (b) the first Brillouin zone, and (c) a nanotube.

with

$$
h_{K}(\hat{\mathbf{k}})=\gamma\left(\hat{k}_{x}-i \hat{k}_{y}\right)+\frac{\beta a \gamma}{4 \sqrt{3}} e^{3 i \eta}\left(\hat{k}_{x}+i \hat{k}_{y}\right)^{2},
$$

where $\gamma$ is a band parameter, $\vec{\sigma}=\left(\sigma_{x}, \sigma_{y}\right)$ are the Pauli spin matrices, $\hat{\mathbf{k}}=\left(\hat{k}_{x}, \hat{k}_{y}\right) \equiv-i \vec{\nabla}$ is a wave-vector operator, $a=2.46 \AA$ is the lattice constant, $\eta$ is a chiral angle between the horizontal axis and the chiral vector $\mathbf{L}$ in Fig. 1(a), which defines the circumference of the nanotube, and $\beta$ and $S$ are dimensionless parameters characterizing trigonal warping of energy bands and electron-hole asymmetry, respectively. In a nearest-neighbor tight-binding model, $\beta=1 .^{30,35}$

Electronic states for a nanotube with a sufficiently large diameter are obtained by imposing a boundary condition in the circumference direction. For the $K$ point, it becomes ${ }^{30,44}$

$$
\mathbf{F}_{K}(\mathbf{r}+\mathbf{L})=\mathbf{F}_{K}(\mathbf{r}) \exp \left[2 \pi i\left(-\frac{v}{3}+\varphi_{K}\right)\right],
$$

with $v$ being an integer determined uniquely as $v=0$ or \pm 1 through $n_{a}+n_{b}=3 M+v$ with integer $M$, where $n_{a}$ and $n_{b}$ are integers defined by $\mathbf{L}=n_{a} \mathbf{a}+n_{b} \mathbf{b}$ [a and $\mathbf{b}$ are the primitive translation vectors shown in Fig. 1(a)], and $\varphi_{K}$ being an effective magnetic flux threading the tube cross section that describes effects of curvature and lattice distortion. The effective magnetic flux is given by ${ }^{32,36-40}$

$$
\varphi_{K}=-\frac{2 \pi}{4 \sqrt{3}} \frac{a}{L} p \cos 3 \eta,
$$

where $L=|\mathbf{L}|$ and $p$ is a dimensionless parameter satisfying $-0.5 \lesssim p \lesssim 0$.

Then, an eigenfunction of Eq. (1) with Eq. (4) is given by a plain wave, which is written as

$$
\mathbf{F}_{K}(\mathbf{r})=\frac{1}{\sqrt{A L}} \mathbf{F}_{\alpha}^{K} e^{i \kappa_{v, n}^{K} x+i k y},
$$

where

$$
\kappa_{v, n}^{K}=\frac{2 \pi}{L}\left(n-\frac{v}{3}+\varphi_{K}\right),
$$

with $A$ being the tube length, $n$ being an integer corresponding to a band index, $k$ being a wave vector in the axis direction, and $\alpha$ being a set of quantum numbers $\alpha=( \pm, n, k)$, where the + and - correspond to conduction and valence bands, respectively. ${ }^{7}$ The vector $\mathbf{F}_{\alpha}^{K}$ is given by

$$
\mathbf{F}_{ \pm, n, k}^{K}=\frac{1}{\sqrt{2}}\left(\begin{array}{c}
b_{v}^{K}(n, k) \\
\pm 1
\end{array}\right)
$$

with

$$
b_{v}^{K}(n, k)=\frac{h_{K}\left(\kappa_{v, n}^{K}, k\right)}{\left|h_{K}\left(\kappa_{v, n}^{K}, k\right)\right|},
$$

and the corresponding eigenenergy is given by

$$
\varepsilon_{ \pm, n, k}^{K}=\frac{\sqrt{3} a \gamma S}{2}\left[\left(\kappa_{v, n}^{K}\right)^{2}+k^{2}\right] \pm\left|h_{K}\left(\kappa_{v, n}^{K}, k\right)\right| .
$$

It can be seen from Eq. (7) that effects of curvature and lattice distortion appear as a shift of the wave vector in the circumference direction.

For the $K^{\prime}$ point, the Hamiltonian matrix is given by the complex conjugate of $\mathcal{H}_{K}$ after replacing $\beta$ with $-\beta$, i.e., $h_{K^{\prime}}(\hat{\mathbf{k}})$ is $h_{K}(\hat{\mathbf{k}})^{\dagger}$ with $\beta \rightarrow-\beta$. The boundary condition for $\mathbf{F}_{K^{\prime}}(\mathbf{r})$ is given by Eq. (4) with replacement of $v$ and $\varphi_{K}$ by $-v$ and $\varphi_{K^{\prime}}=-\varphi_{K}$, respectively. As a result, $\kappa_{v, n}^{K^{\prime}}$ is given by Eq. (7) with replacement of $v \rightarrow-v$ and $\varphi_{K} \rightarrow \varphi_{K^{\prime}}$. Then, $\mathbf{F}_{\alpha}^{K^{\prime}}$ and $\varepsilon_{ \pm, n, k}^{K^{\prime}}$ are given by Eqs. (8) and (9) and Eq. (10), respectively, with $h_{K}\left(\kappa_{v, n}^{K}, k\right) \rightarrow h_{K^{\prime}}\left(\kappa_{v, n}^{K^{\prime}}, k\right)$.

In the absence of the higher-order corrections, tubes with $v=0$ are metallic because $\kappa_{0,0}^{K}=\kappa_{0,0}^{K^{\prime}}=0$ and linear dispersions cross at the Fermi energy, while those with $v= \pm 1$ are semiconducting due to $\kappa_{n, k}^{K} \neq 0$ and $\kappa_{n, k}^{K^{\prime}} \neq 0$ for any $n$ and $k$. Thus, only tubes with $v= \pm 1$ are considered in the following.

Cross-polarized-light absorption causes momentum transfer $2 \pi \hbar l / L$ in the circumference direction where $l$ is equal to the band-index change $\Delta n= \pm 1$ between valence and conduction bands. ${ }^{23,24}$ The corresponding interband transition is that from valence band $n$ to conduction band $n+l$ at a wave vector $k$ both for the $K$ and $K^{\prime}$ valleys. Because the $K$ and $K^{\prime}$ points are converted into each other by a time-reversal operation, ${ }^{46,47}$ states of two valleys are degenerate in the absence of a magnetic field. Thus, the excitation energy is the same as that for the transition from $-n$ to $-n-l$ at $-k$ at the other valley corresponding to momentum transfer $-2 \pi \hbar l / L$. In the presence of the electron-hole symmetry, the energy is equivalent to that for the transition from $-n-l$ to $-n$ at $-k$ with the momentum transfer $2 \pi \hbar l / L$. Therefore, excitation energies for $S=0$ are degenerate between the $K$ and $K^{\prime}$ points for each $l$.

The band parameter $\gamma$ is related to the resonance integral $-\gamma_{0}$ for $\pi$ orbital between the nearest-neighbor sites in a tight-binding model through $\gamma=\sqrt{3} \gamma_{0} a / 2$. Previous studies revealed that $\gamma_{0} \approx 2.7 \mathrm{eV}(\gamma \approx 5.8 \mathrm{eVA})$ can well reproduce experiments. ${ }^{9,48}$ For parameters of the higher-order corrections, it was shown that values around $\beta=1.5$ and $p=-0.2$ or $\beta=1.0$ and $p=-0.5$ reproduce experimental family patterns for parallel polarization reasonably well. ${ }^{7}$ Thus, results for $\beta=1.5$ and $p=-0.2$ will be shown in Sec. III when they are considered. In a tight-binding model, the parameter of the electron-hole asymmetry $S$ arises due to effects of overlapping integral, next-nearest-neighbor interaction, etc. ${ }^{7}$ For graphite, for example, $S \sim 0.13$ has usually been assumed. ${ }^{49}$ 


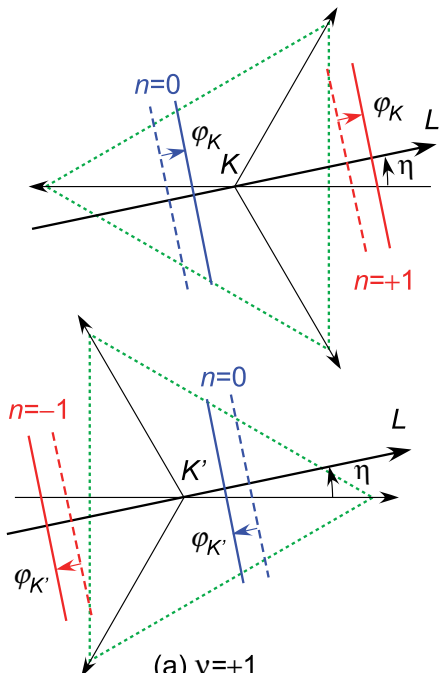

(a) $v=+1$

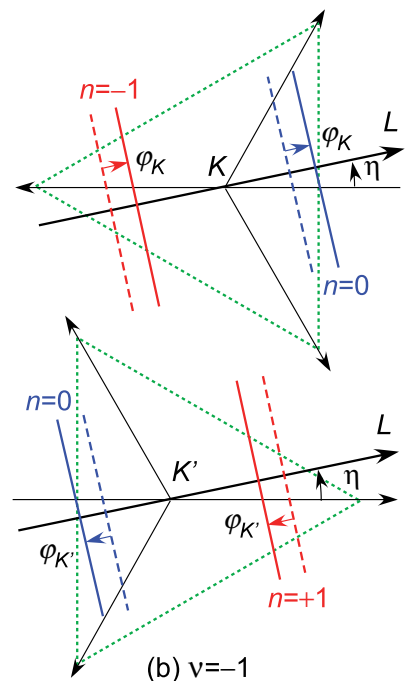

(b) $v=-1$

FIG. 2. (Color online) Schematic illustration of trigonal warping and cutting lines corresponding to two conduction and valence bands close to the $K$ and $K^{\prime}$ points. A shift due to curvature and associated lattice distortion is denoted by effective flux $\varphi_{K}$ and $\varphi_{K^{\prime}}$.

The structure of a nanotube is uniquely specified by chiral vector $\mathbf{L}=n_{a} \mathbf{a}+n_{b} \mathbf{b}$, i.e., by a pair of integers $\left(n_{a}, n_{b}\right)$. Since other indices $\left(n_{1}, n_{2}\right)$ are conventionally used instead of $\left(n_{a}, n_{b}\right)$ where $\left(n_{1}, n_{2}\right)=\left(n_{a}-n_{b}, n_{b}\right)$, we also use $\left(n_{1}, n_{2}\right)$. A family index is defined by $f=2 n_{a}-n_{b}=2 n_{1}+n_{2}$. For tubes with the same family index, projection of $\mathbf{L}$ onto $\mathbf{a}$ is the same, meaning that the circumference lengths are similar.

\section{B. Higher-order effects}

For a tube with $v=+1$, the relevant transition for perpendicular polarization with $\Delta n=1$ is from the valence band with $n=0(n=-1)$ to the conduction band with $n=1$ $(n=0)$ at the $K\left(K^{\prime}\right)$ point. The transition with $\Delta n=-1$ arises between the valence band with $n=1(n=0)$ and the conduction band with $n=0(n=-1)$ at the $K\left(K^{\prime}\right)$ point. For a tube with $v=-1$, relevant transitions are obtained by exchanging the band indices between the $K$ and $K^{\prime}$ points.

Figure 2 shows schematic illustrations of cutting lines corresponding to energy bands in a nanotube in the vicinity of the $K$ and $K^{\prime}$ points [Fig. 2(a) for $v=+1$ and Fig. 2(b) for -1$]$. Dotted lines illustrate equienergy lines with trigonal warping. Arrows labeled by $\mathbf{L}$ indicate direction of the chiral vector with chiral angle $\eta$. Solid and dashed lines are cutting lines for nanotubes with and without curvature and lattice distortion, respectively. The band edges are located at intersection points between the arrows with $\mathbf{L}$ and the cutting lines.

Let us first consider a nanotube with $v=+1$. The trigonal warping tends to decrease the first gap $n=0$ corresponding to the top of the valence band and the bottom of the conduction band and increase the second gap $n= \pm 1$ both for the $K$ and $K^{\prime}$ points. The curvature and lattice distortion, described by $\varphi_{K}$ and $\varphi_{K^{\prime}}$, tend to enhance this tendency. For a nanotube with $v=-1$, on the other hand, the trigonal warping tends to increase the first gap $n=0$ and decrease the second gap

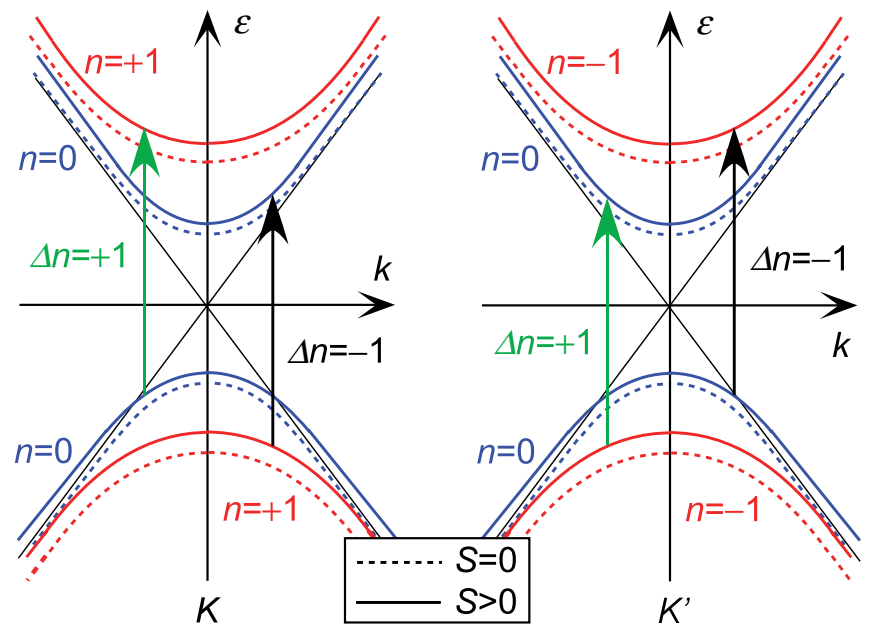

FIG. 3. (Color online) Schematic illustration of effects of asymmetry parameter $S$ on energy bands and optical transitions in the cross-polarization geometry.

$n= \pm 1$, and the curvature and lattice distortion again tend to enhance this tendency. As a result, we have a strong family behavior opposite between the first and second gaps for the parallel polarization characterized by $\Delta n=0$. For cross polarization characterized by $\Delta n= \pm 1$, these effects tend to cancel each other between the first and second bands, making the family effect much weaker.

Because the trigonal warping is stronger for $n= \pm 1$ than for $n=0$ due to larger distance from the $K$ and $K^{\prime}$ points, its effects on excitation energy for perpendicular polarization are dominated by the bands with $n= \pm 1$. Effects of nonzero $\varphi_{K}$ and $\varphi_{K^{\prime}}$ almost cancel each other between $n= \pm 1$ and $n=0$. Therefore, the excitation energies for the cross-polarization exhibit very weak family behavior governed by that of the second gap $n= \pm 1$. On the other hand, the reduced effective mass, playing important roles in the exciton binding, is dominated by the first band $n=0$ with a smaller effective mass; therefore, the family behavior of the oscillator strength is determined by that of the first gap, exhibiting the behavior similar to that of $n=0$ for parallel polarization.

The asymmetry of electrons and holes causes energy shift proportional to the square of the length of a wave vector as shown in Eq. (10). Figure 3 schematically shows energy bands of a tube with $v=+1$ for $S>0$ (solid lines) and $S=0$ (dashed lines). The electron-hole asymmetry lifts the degeneracy of interband transition energies between the $K$ and $K^{\prime}$ points present for $S=0$. The transition energies for $\Delta n=1$ are given for a tube with $v=+1$ by $\varepsilon_{+, 1, k}^{K}-\varepsilon_{-, 0, k}^{K}=$ $\Delta \varepsilon_{k}+\Delta$ and $\varepsilon_{+, 0, k}^{K^{\prime}}-\varepsilon_{-,-1, k}^{K^{\prime}}=\Delta \varepsilon_{k}-\Delta$ for the $K$ and $K^{\prime}$ points, respectively, where

$$
\begin{gathered}
\Delta \varepsilon_{k}=\left|h_{K}\left(\kappa_{1,1}^{K}, k\right)\right|+\left|h_{K}\left(\kappa_{1,0}^{K}, k\right)\right| \\
=\left|h_{K^{\prime}}\left(\kappa_{1,0}^{K^{\prime}}, k\right)\right|+\left|h_{K^{\prime}}\left(\kappa_{1,-1}^{K^{\prime}}, k\right)\right|, \\
\Delta=\frac{2 \pi^{2} a \gamma S}{\sqrt{3} L^{2}} .
\end{gathered}
$$

Figures 4(a) and 4(b) show typical examples of calculated energy bands near the $K$ point for $v=+1$ and -1 , 

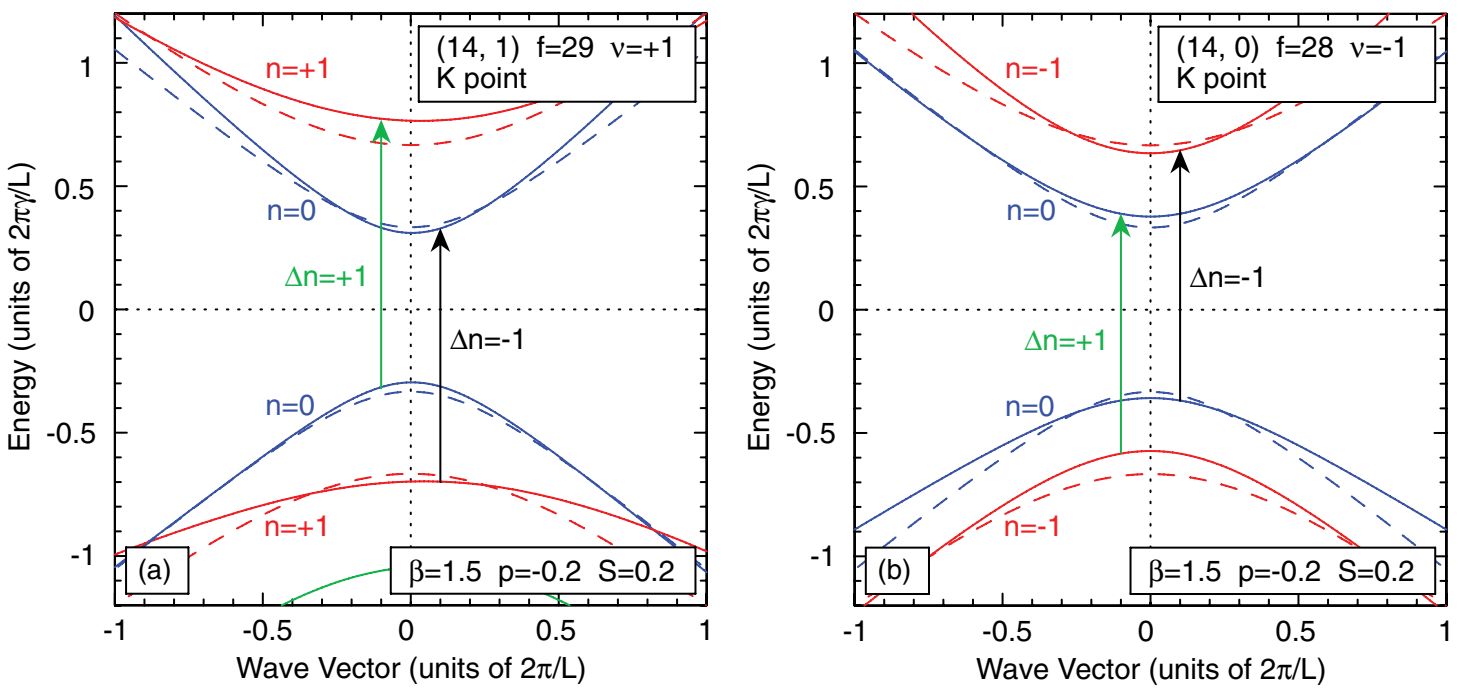

FIG. 4. (Color online) Energy bands around the $K$ point of (a) a $(14,1)$ tube with $v=+1$ and (b) a $(14,0)$ tube with $v=-1$ for $\beta=1.5$, $p=-0.2$, and $S=0.2$. Dashed lines denote results for $\beta=p=S=0$.

respectively. Solid lines denote bands in the presence of higher-order terms with $\beta=1.5, p=-0.2$, and $S=0.2$, and dashed lines denote those without higher-order terms, i.e., $\beta=p=S=0$. Higher-order terms cause the behavior due to the effects discussed in this paper. It is noted that the electron-hole asymmetry is independent of the chiral angle and, therefore, family behavior mainly comes from trigonal warping, curvature, and lattice distortion.

\section{Exciton}

In calculations of interaction effects on the band structure, a screened Hartree-Fock approximation is used and attractive interaction between a photoexcited electron and a remaining hole is introduced by using the Coulomb interaction screened by a static dielectric function. ${ }^{8,9,50}$ This scheme was shown to be sufficient by comparison with results of calculations in a higher GW-type approximation. ${ }^{51,52}$

An exciton wave function at each valley with momentum $2 \pi \hbar l / L$ in the circumference direction is written as

$$
|u, l\rangle=\sum_{n, k} \psi_{n}^{l}(k) c_{+, n+l, k}^{\dagger} c_{-, n, k}|g\rangle,
$$

where $c_{\alpha, k}^{\dagger}$ and $c_{\alpha, k}$ are the creation and annihilation operators for electrons, respectively, and $|g\rangle$ is the ground state. The coefficients $\psi_{n}^{l}(k)$ and energy eigenvalues for the corresponding states are obtained by solving an equation of motion for an electron and a hole under attractive interaction. ${ }^{8,9,50}$ Weak short-range parts of the Coulomb interaction causing coupling between the $K$ and $K^{\prime}$ points ${ }^{17,21,50,53}$ are completely neglected because they are about a few millielectronvolts ${ }^{54-62}$ and much smaller than intervalley interaction for the depolarization effects of the order of a few hundred millielectronvolts. ${ }^{21,25-29}$

A dielectric constant $\kappa$ is introduced to describe effects of screening by electrons in $\sigma$ bands, core states, and the $\pi$ bands away from the $K$ and $K^{\prime}$ points. The interaction strength is then characterized by a dimensionless parameter $\left(e^{2} / \kappa L\right)(2 \pi \gamma / L)^{-1}$, which is the ratio be- tween a typical Coulomb interaction and a typical kinetic energy. Previous analysis of excitation energies has shown that $0.1 \lesssim\left(e^{2} / \kappa L\right)(2 \pi \gamma / L)^{-1} \lesssim 0.2 .^{9,48}$ Therefore, $\left(e^{2} / \kappa L\right)(2 \pi \gamma / L)^{-1}=0.15$ is used in the following.

\section{Dynamical conductivity}

The dynamical conductivity characterizing optical absorption is calculated by using the Kubo formula. ${ }^{63}$ The velocity operator in the circumference direction is given by

$$
v_{x}(\hat{\mathbf{k}})=\frac{1}{\hbar} \frac{\partial \mathcal{H}}{\partial \hat{k}_{x}} .
$$

Then, for the $K$ point, we have

$$
v_{x}^{K}(\hat{\mathbf{k}})=\frac{\gamma}{\hbar}\left(\begin{array}{ll}
\sqrt{3} a S \hat{k}_{x} & V_{x}^{K}(\hat{\mathbf{k}}) \\
V_{x}^{K}(\hat{\mathbf{k}})^{\dagger} & \sqrt{3} a S \hat{k}_{x}
\end{array}\right),
$$

with

$$
V_{x}^{K}(\hat{\mathbf{k}})=1+\frac{\beta a}{2 \sqrt{3}} e^{3 i \eta}\left(\hat{k}_{x}+i \hat{k}_{y}\right) .
$$

For the $K^{\prime}$ point, $v_{x}^{K^{\prime}}(\hat{\mathbf{k}})$ is given by Eq. (15) with replacement of $V_{x}^{K}(\hat{\mathbf{k}}) \rightarrow V_{x}^{K^{\prime}}(\hat{\mathbf{k}})$, where $V_{x}^{K^{\prime}}(\hat{\mathbf{k}})$ is the complex conjugate of Eq. (16) with $\beta \rightarrow-\beta$.

The $x$ component of the velocity operator for wave vector $2 \pi l / L$ in the circumference direction is given by

$$
\hat{v}_{x, l}=\frac{1}{2}\left[v_{x}(\hat{\mathbf{k}}) e^{i l \hat{\theta}}+e^{i l \hat{\theta}} v_{x}(\hat{\mathbf{k}})\right],
$$

with $\hat{\theta}=2 \pi \hat{x} / L$ [where $\hat{x}$ is the position operator in the circumference direction]. Its matrix element between the ground state and the exciton state is

$$
\left\langle u, l\left|\hat{v}_{x, l}\right| g\right\rangle=\sum_{n, k}\left(v_{x, l}\right)_{n, k} \psi_{n}^{l^{*}}(k),
$$

where $\left(v_{x, l}\right)_{n, k}$ is a matrix element of $\hat{v}_{x, l}$ between the oneparticle state with $\kappa_{v, n+l}$ and $k$ at the conduction band and the 
one-particle state with $\kappa_{v, n}$ and $k$ at the valence band. For the $K$ point, the matrix element is given by

$$
\left(v_{x, l}^{K}\right)_{n, k}=\mathbf{F}_{+, n+l, k}^{K \dagger} v_{x}^{K}\left(\kappa_{v, n+l / 2}^{K}, k\right) \mathbf{F}_{-, n, k}^{K} .
$$

For the $K^{\prime}$ point, the matrix element is given by the replacement of $\mathbf{F}_{\alpha}^{K}$ and $v_{x}^{K}\left(\kappa_{v, n+l / 2}^{K}, k\right)$ with $\mathbf{F}_{\alpha}^{K^{\prime}}$ and $v_{x}^{K^{\prime}}\left(\kappa_{v, n+l / 2}^{K^{\prime}}, k\right)$, respectively.

For cross-polarized light with momentum $2 \pi \hbar l / L$ in the circumference direction, the associated dynamical conductivity is given by

$$
\sigma_{x x}^{l}(\omega)=\frac{g_{s} \hbar e^{2}}{A L} \sum_{K K^{\prime}} \sum_{u} \frac{-2 i \hbar \omega\left|\left\langle u, l\left|\hat{v}_{x, l}\right| g\right\rangle\right|^{2}}{\varepsilon_{u}\left[\varepsilon_{u}^{2}-(\hbar \omega)^{2}-2 i \hbar \omega \Gamma\right]},
$$

where $g_{s}=2$ represents the spin degeneracy and a phenomenological energy broadening $\Gamma$ has been introduced.

For cross-polarized light, the depolarization effect, in which the electric field associated with induced polarization arises, ${ }^{23,24}$ must be considered. It is taken into account in a self-consistent-field method where an optically induced current and electric field that electrons feel are determined self-consistently. ${ }^{23,24,64-66}$ Then, the absorption power is proportional to the real part of $\tilde{\sigma}_{x x}(\omega)$, defined by

$$
\tilde{\sigma}_{x x}(\omega)=\frac{1}{2}\left[\tilde{\sigma}_{x x}^{l=1}(\omega)+\tilde{\sigma}_{x x}^{l=-1}(\omega)\right],
$$

where

$$
\tilde{\sigma}_{x x}^{l}(\omega)=\frac{\sigma_{x x}^{l}(\omega)}{\varepsilon_{x x}^{l}(\omega)}
$$

with

$$
\varepsilon_{x x}^{l}(\omega)=1+\frac{4 \pi^{2} i}{\kappa L \omega} \sigma_{x x}^{l}(\omega) .
$$

In this case, excitation energies are given by zero points $\omega_{0}$ of $\varepsilon_{x x}^{l}(\omega)$, i.e., $\varepsilon_{x x}^{l}\left(\omega_{0}\right)=0$. According to Eq. (20), in the limit of $\Gamma \rightarrow 0$, the real part of $\varepsilon_{x x}^{l}(\omega)$ changes from $-\infty$ to $\infty$ between each pair of neighboring excitation energies $\varepsilon_{u}$ 's, while the imaginary part is zero. Then, $\omega_{0}$ is located in each interval between the neighboring $\varepsilon_{u}$ 's. The intensity of $\operatorname{Re}\left[\tilde{\sigma}_{x x}^{l}(\omega)\right]$ at $\omega_{0}$ is proportional to the inverse of the derivative of $\varepsilon_{x x}^{l}(\omega)$ with respect to $\omega$ as described in the next section.

\section{E. Brightening of the dark exciton}

Excitation energies without the depolarization effect $\left[\varepsilon_{u}\right.$ 's in Eq. (20)] are degenerate between the $K$ and $K^{\prime}$ points when the electron-hole symmetry exists as described in Sec. II A. There is no optically allowed exciton in the vicinity of $\varepsilon_{u}$. In the presence of electron-hole asymmetry, the lifting of the degeneracy induces an optically allowed exciton with small oscillator strength between these split energy levels. This is similar to the brightening due to the Aharonov-Bohm splitting between the $K$ and $K^{\prime}$ excitons by magnetic field. ${ }^{42,50}$

When we consider the energy region close to $\varepsilon_{u}$ of the lowest exciton, we approximately have

$$
\begin{aligned}
\sigma_{x x}^{l}(\omega)= & \frac{g_{s} g_{v} \hbar e^{2}\left|\left\langle u, l\left|\hat{v}_{x, l}\right| g\right\rangle\right|^{2}}{A L \varepsilon_{u}} \frac{1}{2}\left[\frac{-2 i \hbar \omega}{\left(\varepsilon_{u}-\Delta\right)^{2}-(\hbar \omega)^{2}}\right. \\
& \left.+\frac{-2 i \hbar \omega}{\left(\varepsilon_{u}+\Delta\right)^{2}-(\hbar \omega)^{2}}\right],
\end{aligned}
$$

where $g_{v}=2$ is the valley degeneracy, $\left\langle u, l\left|\hat{v}_{x, l}\right| g\right\rangle$ is a velocity matrix element for the lowest exciton without the depolarization effects for $S=0$, and $\Delta$ is energy splitting due to $S$ given by Eq. (12). Correspondingly, we have

$\varepsilon_{x x}^{l}(\omega)=1+\frac{1}{2}\left[\frac{\left(\hbar \omega_{p}\right)^{2}}{\left(\varepsilon_{u}-\Delta\right)^{2}-(\hbar \omega)^{2}}+\frac{\left(\hbar \omega_{p}\right)^{2}}{\left(\varepsilon_{u}+\Delta\right)^{2}-(\hbar \omega)^{2}}\right]$,

with

$$
\left(\hbar \omega_{p}\right)^{2}=\frac{8 g_{s} g_{v} \pi^{2} \hbar^{2} e^{2}\left|\left\langle u, l\left|\hat{v}_{x, l}\right| g\right\rangle\right|^{2}}{\kappa A L^{2} \varepsilon_{u}} .
$$

The zero points of $\varepsilon_{x x}^{l}(\omega)$ are obtained up to the second order in $\Delta / \varepsilon_{u}$ as

$$
\begin{gathered}
\left(\frac{\hbar \omega_{-}}{\varepsilon_{u}}\right)^{2}=1-\left(\frac{\Delta}{\varepsilon_{u}}\right)^{2}\left[\left(\frac{2 \varepsilon_{u}}{\hbar \omega_{p}}\right)^{2}-1\right], \\
\left(\frac{\hbar \omega_{+}}{\varepsilon_{u}}\right)^{2}=1+\left(\frac{\hbar \omega_{p}}{\varepsilon_{u}}\right)^{2}+\left(\frac{\Delta}{\varepsilon_{u}}\right)^{2}\left[\left(\frac{2 \varepsilon_{u}}{\hbar \omega_{p}}\right)^{2}+1\right] .
\end{gathered}
$$

The low-frequency solution $\omega_{-}$corresponds to the excitation energy of the brightened dark exciton and the high-frequency solution $\omega_{+}$corresponds to that of the depolarization-shifted bright exciton. This shows that the energy of the brightened dark exciton becomes slightly smaller than $\varepsilon_{u}$ because $\hbar \omega_{p}$ is usually smaller than $\varepsilon_{u}$.

The conductivity including the depolarization effect is given by

$$
\begin{aligned}
\tilde{\sigma}_{x x}^{l}(\omega)= & \frac{g_{s} g_{v} \hbar e^{2}\left|\left\langle u, l\left|\hat{v}_{x, l}\right| g\right\rangle\right|^{2}\left[\varepsilon_{u}^{2}+\Delta^{2}-(\hbar \omega)^{2}\right]}{A L \varepsilon_{u}\left[2 \varepsilon_{u}^{2}+2 \Delta^{2}+\left(\hbar \omega_{p}\right)^{2}-2(\hbar \omega)^{2}\right]} \\
& \times\left[\frac{-2 i \hbar \omega}{\left(\hbar \omega_{-}\right)^{2}-(\hbar \omega)^{2}}+\frac{-2 i \hbar \omega}{\left(\hbar \omega_{+}\right)^{2}-(\hbar \omega)^{2}}\right] .
\end{aligned}
$$

This is written near $\omega=\omega_{-}$as

$$
\tilde{\sigma}_{x x}^{l}(\omega) \approx \frac{4 \varepsilon_{u}^{2} \Delta^{2}}{\left(\hbar \omega_{p}\right)^{4}} \frac{g_{s} g_{v} \hbar e^{2}\left|\left\langle u, l\left|\hat{v}_{x, l}\right| g\right\rangle\right|^{2}}{A L \varepsilon_{u}} \frac{-2 i \hbar \omega}{\left(\hbar \omega_{-}\right)^{2}-(\hbar \omega)^{2}},
$$

which shows that the intensity is reduced by factor $4 \varepsilon_{u}^{2} \Delta^{2} /\left(\hbar \omega_{p}\right)^{4}$ from that of the absorption peak for excitons at $\varepsilon_{u}$ without the depolarization effect. Near $\omega=\omega_{+}$, on the other hand, we have

$$
\begin{aligned}
\tilde{\sigma}_{x x}^{l}(\omega) & \approx\left[1-\frac{4 \varepsilon_{u}^{2} \Delta^{2}}{\left(\hbar \omega_{p}\right)^{4}}\right] \\
& \times \frac{g_{s} g \hbar \hbar e^{2}\left|\left\langle u, l\left|\hat{v}_{x, l}\right| g\right\rangle\right|^{2}}{A L \varepsilon_{u}} \frac{-2 i \hbar \omega}{\left(\hbar \omega_{+}\right)^{2}-(\hbar \omega)^{2}},
\end{aligned}
$$

which shows that the intensity is slightly reduced in the presence of asymmetry splitting $\Delta$.

Actually, this estimation of the intensity and resonance position is not valid for $\omega_{+}$, but it is quite accurate for $\omega_{-}$. The reason lies in the presence of excited exciton states and the interband continuum. In fact, the $\hbar \omega_{+}$given by Eq. (28) lies in the energy region of the interband continuum and therefore is pushed down to a position lower than, but close to, the first excited exciton. As a result, the intensity is considerably suppressed due to enhancement of the energy derivative of 


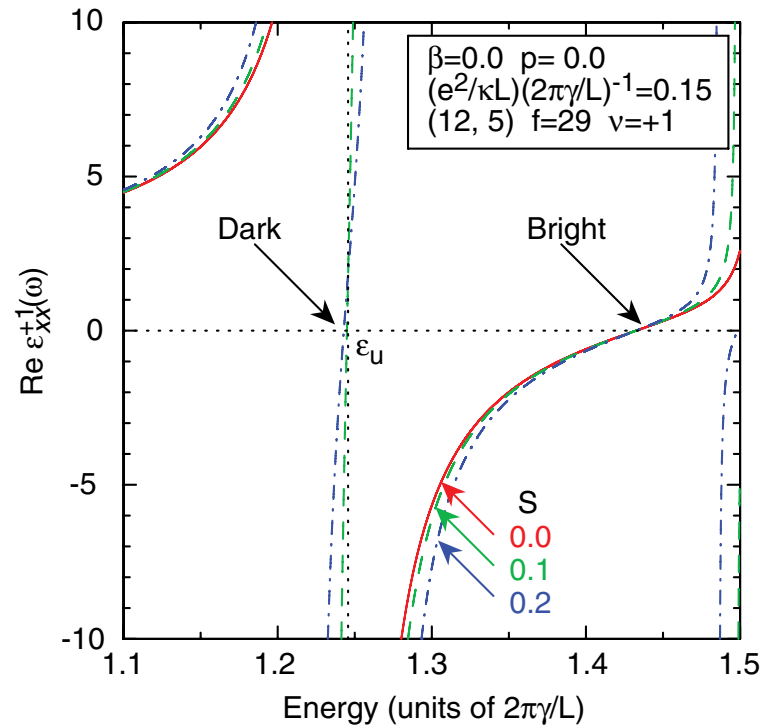

FIG. 5. (Color online) Real part of $\varepsilon_{x x}^{+1}(\omega)$ for $\beta=p=0$ as a function of $\hbar \omega$. The results for $S=0,0.1$, and 0.2 are shown.

$\varepsilon_{x x}^{l}(\omega)$. Previous calculations for $\Delta=0$ showed that the intensity is strongly reduced from that given by Eq. (31). ${ }^{25,42}$

Figure 5 shows an example of the real part of $\varepsilon_{x x}^{l}(\omega)$ as a function of energy $\hbar \omega$ around energy $\varepsilon_{u}$ of the lowest exciton. In this figure, we have completely neglected higher-order terms except $S$. With the increase of $S$, a zero point corresponding to brightening of the dark exciton appears in the vicinity of $\varepsilon_{u}$ and is slightly shifted to the lower energy side in agreement with Eq. (27). The energy of the bright exciton lying in the higher energy side remains essentially independent of $S$ below the first excited exciton state in contrast to Eq. (28). Further, the energy derivative of $\varepsilon_{x x}^{l}$ at the zero point becomes larger with $S$ due to the lowering of excited exciton states, leading to a slight reduction in the oscillator strength with increasing $S$, as will be demonstrated in numerical results presented in the next section.

\section{NUMERICAL RESULTS}

The effects of parameter $S$ are considered and higher-order corrections leading to the family behavior are completely neglected, i.e., $\beta=p=0$, for the purpose of clarifying roles of the electron-hole asymmetry. Figure 6 shows typical dynamical conductivity for $S=0$ (solid line), 0.1 (dashed line), and 0.2 (dashed-dotted line). For $S=0$ a main peak appears at $\varepsilon(2 \pi \gamma / L)^{-1} \approx 1.43$ as well as a small peak at 1.53, coming from the lowest and the excited bright excitons. When $S$ is increased, the position of the main peak remains the same, but its intensity gradually decreases.

Further, a new peak appears at $\varepsilon(2 \pi \gamma / L)^{-1} \approx 1.24$ with the increase of $S$, corresponding to brightening of dark excitons due to the electron-hole asymmetry as described in Sec. IIE. With the increase of $S$, the position of this peak is slightly shifted to the lower energy side and the intensity increases. A smaller peak appears below the excited bright exciton $\left[\varepsilon(2 \pi \gamma / L)^{-1} \approx 1.5\right.$ for $\left.S=0.1\right]$ and its intensity becomes stronger with $S$. This behavior is nothing but asymmetry-

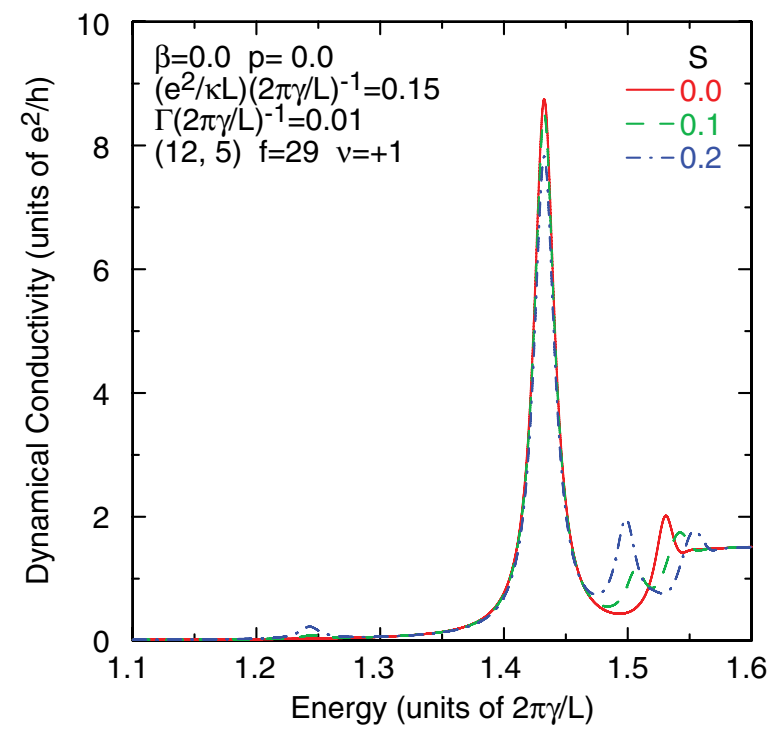

FIG. 6. (Color online) Dynamical conductivity of the (12,5) tube for $S=0$ (solid line), $S=0.1$ (dashed line), and $S=0.2$ (dasheddotted line). $\beta=p=0$.

induced brightening for the first excited exciton, which has also become optically forbidden by the depolarization effect.

Figure 7 shows excitation energies of the lowest bright and the lowest brightened dark excitons as a function of the circumference length. The former and latter are denoted by bright and dark, respectively. The parameter $S$ is chosen as $S=0$ (solid lines), $S=0.1$ (dashed lines), and $S=0.2$ (dashed-dotted lines). The energies are approximately proportional to the inverse of the circumference length. With the increase of $S$, the energy of the brightened dark exciton is slightly reduced, although the change is negligible in the scale of this figure.

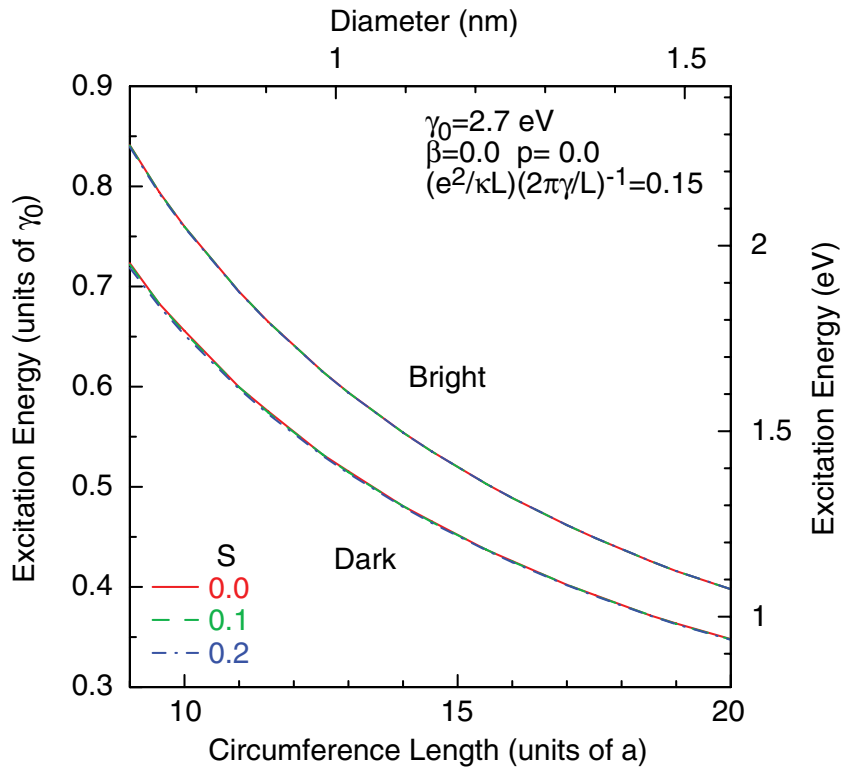

FIG. 7. (Color online) Circumference-length dependence of excitation energy of the bright and the brightened dark excitons for $S=0$ (solid lines), 0.1 (dashed lines), and 0.2 (dashed-dotted lines). 


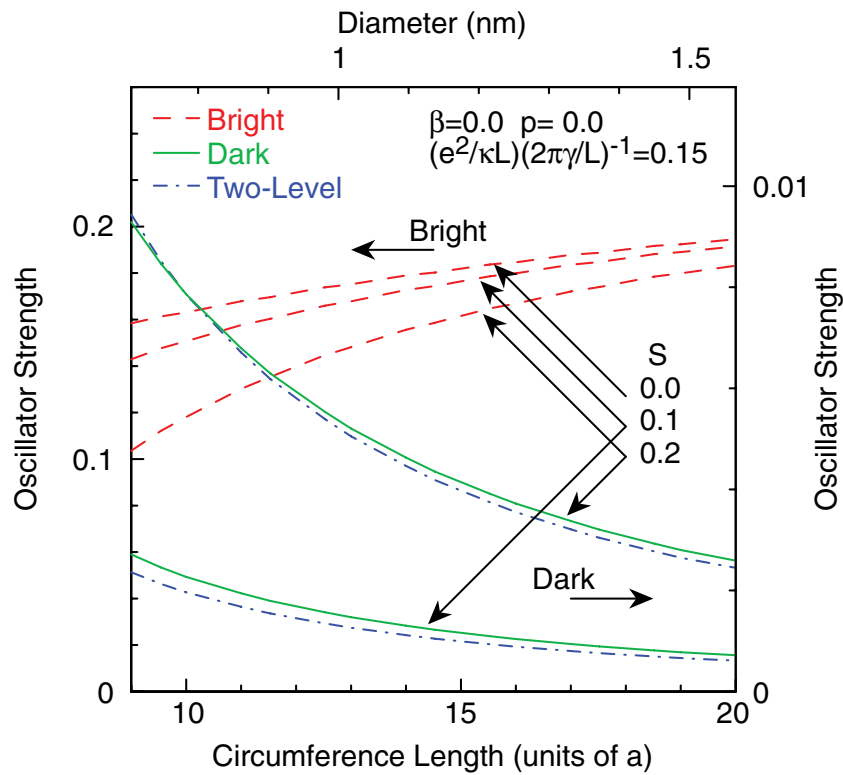

FIG. 8. (Color online) Circumference-length dependence of oscillator strength for the bright exciton (dashed lines) and the brightened dark exciton (solid lines) for $S=0,0.1$, and 0.2. Scales for the bright and the brightened dark excitons are plotted in the left and right vertical axes, respectively. Dashed-dotted lines are the results in the two-level approximation.

In the following, the oscillator strength for an exciton with the depolarization effect at $\hbar \omega_{0}$ will be defined as ${ }^{25}$

$$
f_{0}=2 m^{*} \frac{\kappa L^{3} \omega_{0}}{8 \pi^{2} \hbar e^{2}}\left[\frac{1}{\hbar} \frac{\partial \varepsilon_{x x}^{l}}{\partial \omega}\left(\omega_{0}\right)\right]^{-1},
$$

where the effective mass of the first conduction band is defined by $m^{*}=\left(2 \pi \hbar^{2}\right) /(3 \gamma L)$. Then, the oscillator strength for the lowest brightened dark exciton near $\varepsilon_{u}$ is given in the two-level approximation in Sec. II E by

$$
f_{0}=\frac{\pi^{2} a^{2} S^{2}}{36 L^{2}}\left[\left(\frac{\kappa L}{e^{2}} \frac{2 \pi \gamma}{L}\right)^{2}\left(\frac{\varepsilon_{u} L}{2 \pi \gamma}\right)^{2} \frac{A \gamma \varepsilon_{u}}{2 \pi \hbar^{2}\left|\left\langle u, l\left|\hat{v}_{x, l}\right| g\right\rangle\right|^{2}}\right],
$$

which is approximately proportional to $S^{2}(L / a)^{-2}$ because the dimensionless quantities in the brackets are independent of $L$ apart from the small change through the screening effect to be discussed in the following.

In Fig. 8, the oscillator strength of the lowest bright exciton and that of the lowest brightened dark exciton are shown for $S=0,0.1$, and 0.2. Dashed and solid lines denote the bright and the brightened dark excitons, respectively. The dasheddotted lines denote the results of the two-level approximation given by Eq. (33). For the brightened dark exciton, the oscillator strength is well reproduced by the two-level approximation. For the bright exciton, the oscillator strength slightly increases with an increase of the circumference length. This comes from the increase in screening effects with $L$ and the resulting reduction in the depolarization effect, because the number of bands contributing to the screening increases with $L$.

Next, we consider the parameters $\beta=1.5$ and $p=-0.2$ responsible for family behavior. In Fig. 9, excitation energies

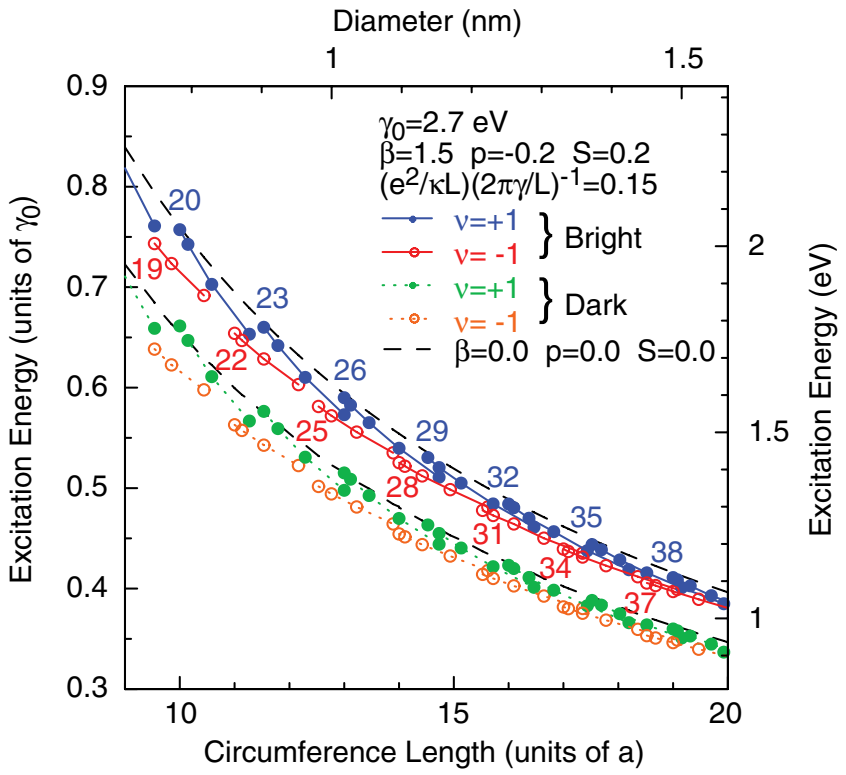

FIG. 9. (Color online) Circumference-length dependence of excitation energy for $S=0.2, \beta=1.5$, and $p=-0.2$. Circles connected by solid and dotted lines indicate results for the bright and the brightened dark excitons, respectively. Closed and open circles indicate $v=+1$ and -1 , respectively. Dashed lines are results for $\beta=p=S=0$.

for the lowest bright and the lowest brightened dark excitons are shown as a function of the circumference length. Closed and open circles denote $v=+1$ and -1 , respectively. Numbers indicate family indices $f$. Although only the results for $S=0.2$ are shown, the dependence of these energies on $S$ is negligible as mentioned before.

The family pattern is much weaker than for parallel polarization. Further, it is qualitatively similar to that of the second gap for parallel polarization, i.e., the upper and lower branches consist of tubes with $v=+1$ and -1 , respectively. This is in agreement with the qualitative discussion presented in Sec. II B, and is also consistent with experiments. $^{28}$

Figure 10 shows the circumference-length dependence of oscillator strength for the lowest bright and the lowest brightened dark excitons. The asymmetry parameter is chosen as $S=0.1$ and 0.2 in Figs. 10(a) and 10(b), respectively. In contrast to the energy, a clear family pattern can be seen in the intensity for both excitons. The upper and lower branches of the family pattern for the bright exciton consist of tubes with $v=-1$ and +1 , respectively, and are essentially determined by effects of $\beta$ and $p$ on the bands $n=0$ as described in Sec. II B.

\section{DISCUSSIONS}

The calculated excitation energies for the lowest bright and the lowest brightened dark excitons are compared with experiments in Fig. 11. Circles denote the present results. For the bright exciton, an empirical formula ${ }^{28}$ is drawn by dashed lines and experiments are plotted by triangles ${ }^{29}$ and open squares. ${ }^{43}$ Crosses are experiments for the brightened dark exciton. ${ }^{43}$ The theory is semiquantitatively in agreement 


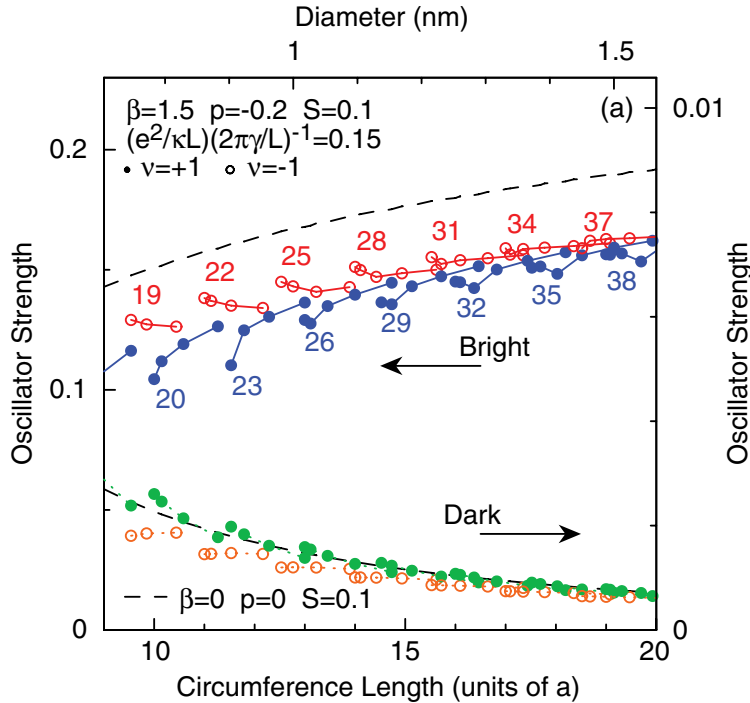

Diameter $(\mathrm{nm})$

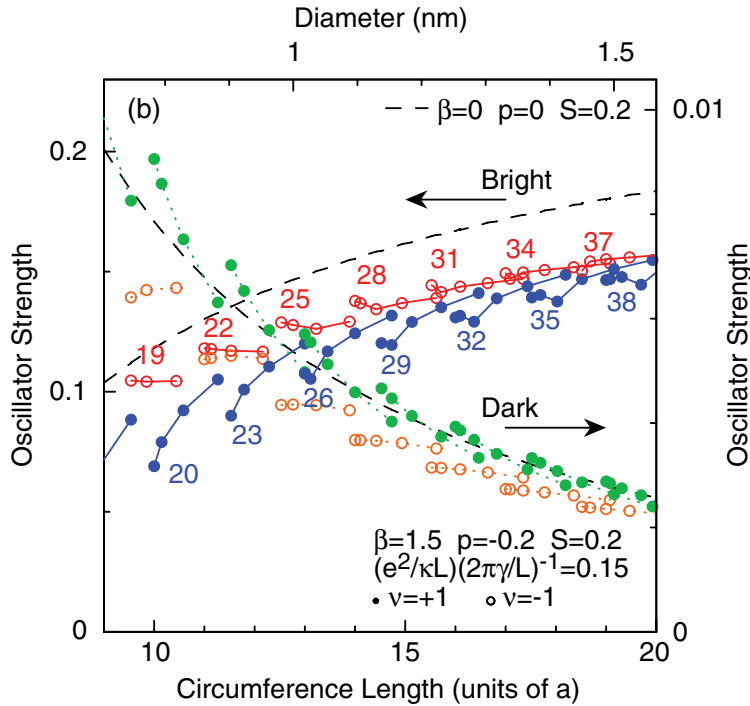

FIG. 10. (Color online) Circumference-length dependence of oscillator strength for the bright and the brightened dark excitons for (a) $S=0.1$ and (b) $S=0.2$. Circles connected by solid and dotted lines indicate results for the bright and the brightened dark excitons, respectively. Scales for the bright and the brightened dark excitons are plotted in the left and right vertical axes, respectively. Dashed lines are results for $\beta=p=0$.

with the experiments. However, more detailed comparison is not feasible yet because of the presence of some deviations among experiments.

In Fig. 12, comparison with experiments is made for the relative intensity of the lowest brightened dark exciton. The intensity is normalized in such a way that the intensity for the lowest brightened dark exciton is divided by the sum of itself and the intensity for the lowest bright exciton. The circles show the intensity for $S=0.2$, obtained by including all higher-order terms $(\beta=1.5$ and $p=-0.2)$, and dashed lines denote results for $\beta=p=0$. Closed and open triangles are experimental results for $v=+1$ and -1 , respectively. ${ }^{43}$ Several experimental results lying around 0.05 can be well reproduced by the theory for $S \approx 0.2$, but other points lying much higher cannot be reproduced even for $S=0.3$.

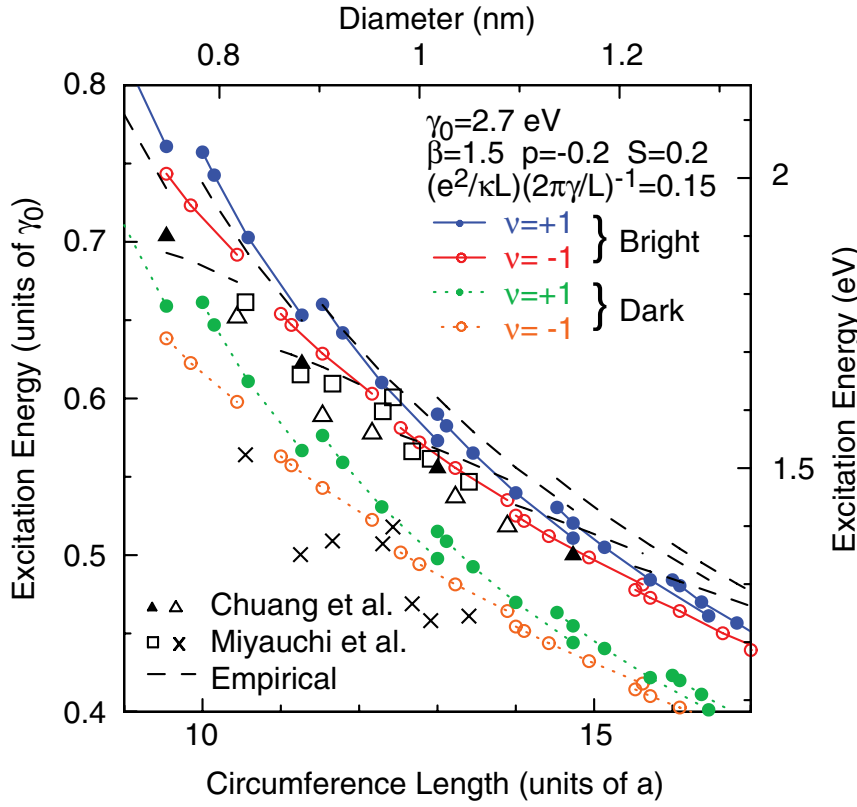

FIG. 11. (Color online) Circumference-length dependence of excitation energy of the bright and the brightened dark excitons. Circles are theory. Dashed lines are empirical, squares and triangles are experiments for the bright exciton, and crosses are experiments for the brightened dark exciton. Closed circles and triangles denote $v=+1$, corresponding open symbols denote $v=-1$, and $v$ for squares is unknown.

As shown in Eq. (33), the intensity of the brightened dark exciton is sensitive to the strength of the Coulomb interaction and increases with the decrease of the interaction strength. However, the strong enhancement observed in experiments is

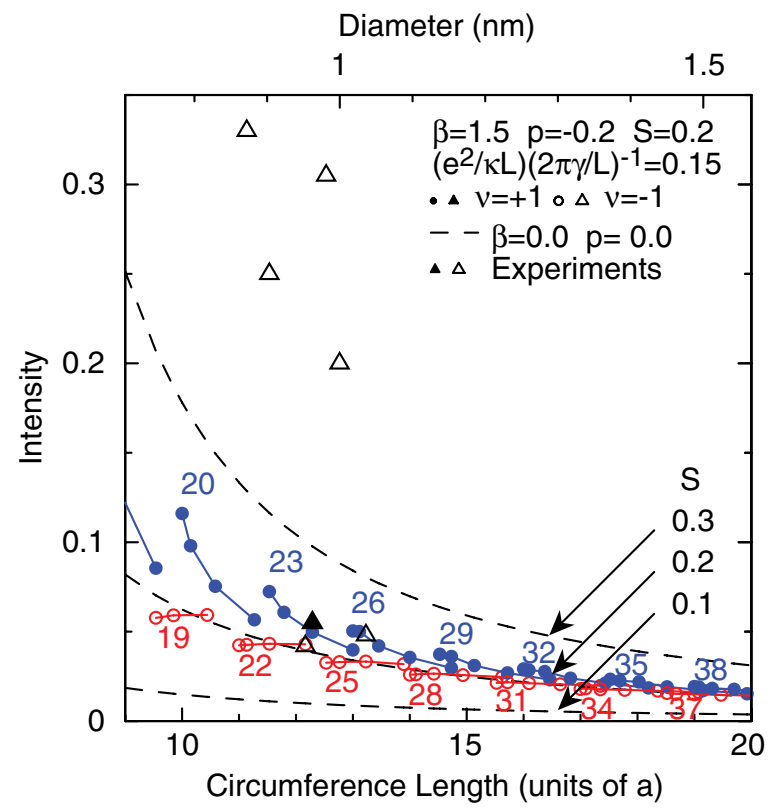

FIG. 12. (Color online) Circumference-length dependence of normalized intensity for the brightened dark exciton. Circles are theory for $\beta=1.5, p=-0.2$, and $S=0.2$ and dashed lines are theory with $\beta=p=0$ for $S=0.1,0.2$, and 0.3 . Triangles are experiments. Closed and open symbols denote $v=+1$ and -1 , respectively. 
not likely to be understood by a slight change in the interaction strength, and, therefore, the reason for the disagreement remains unclear. Because of the strong depolarization effect in the cross-polarization geometry, excitons are likely to be strongly affected by their environment, ${ }^{20,31,67-73}$ giving rise to differences between experimental results. This environment effect may have to be considered to explain the difference.

Brightening of dark excitons due to electron-hole asymmetry was previously discussed based on calculations in a similar effective-mass approximation. ${ }^{43}$ Quantitatively, however, there seem to be some significant differences between these results and those of Ref. 43. In fact, in Ref. 43, the main absorption peak of the lowest bright exciton is shifted to the higher energy side and its intensity remains essentially the same with the increase of $S$. On the contrary, these results show that the peak position remains independent of $S$ and its intensity is slightly reduced with the increase of $S$. Further, the normalized intensity for the lowest brightened dark exciton seems to be larger than these results. A slightly stronger interaction parameter $\left(e^{2} / \kappa L\right)(2 \pi \gamma / L)^{-1}=0.19$ was used in Ref. 43 in contrast to $\left(e^{2} / \kappa L\right)(2 \pi \gamma / L)^{-1}=$ 0.15 used in this paper. This alone can not explain the qualitative and quantitative differences mentioned above.

It is impossible to further pursue the origin of these differences because the method of including the depolarization effect is not explicitly described in Ref. 43. There are various schemes used to treat the depolarization effect. This self-consistent-field method is exact and has, for example, successfully explained intersubband optical transitions in space-charge layers on an $\mathrm{Si}$ surface. ${ }^{64-66}$ This method is reformulated in such a way that the Schrödinger and Maxwell equations for electrons and electromagnetic fields, respectively, are solved self-consistently. ${ }^{74-76}$ It was applied to cross-polarized excitons in carbon nanotubes under a rotatingwave approximation. ${ }^{41,77}$
The Bethe-Salpeter-type equation is often solved with a so-called exchange term, approximately describing the depolarization effect, under the condition that only a single electronhole pair is excited, ${ }^{18,21,26,78,79}$ i.e., in a single configuration interaction approximation equivalent to the Tamm-Dancoff approximation. ${ }^{80}$ Both rotating-wave and Tamm-Dancofftype approximations are certainly valid when the spectrum is dominated by a single resonance peak. In nanotubes in the cross-polarized geometry, however, various resonances coexist and approximate methods can give results significantly different from exact, depending on the degree of approximations.

\section{SUMMARY AND CONCLUSION}

We have investigated effects of higher-order terms in an effective-mass approximation on cross-polarized excitons in semiconducting carbon nanotubes. Electron-hole asymmetry causes the appearance of new peaks in absorption spectra due to brightening of optically forbidden excitons called dark excitons. Family effects on excitation energy are much smaller than those for parallel polarization, and they are more clearly seen in oscillator strength. Calculated excitation energies are in good agreement with experiments. There remains some disagreement in the intensity of some of brightened dark excitons.

\section{ACKNOWLEDGMENTS}

This work was supported in part by a Global Center of Excellence Program at Tokyo Tech "Nanoscience and Quantum Physics," Grants-in-Aid for Scientific Research, and a Grant-in-Aid for Scientific Research on Priority Area "Carbon Nanotube Nanoelectronics" from the Ministry of Education, Culture, Sports, Science, and Technology, Japan. The numerical calculations were partly performed at the Supercomputer Center, Institute for Solid State Physics, University of Tokyo, and at Iwate University Super Computing and Information Sciences Center.
${ }^{1}$ S. M. Bachilo, M. S. Strano, C. Kittrell, R. H. Hauge, R. E. Smalley, and R. B. Weisman, Science 298, 2361 (2002).

${ }^{2}$ R. B. Weisman and S. M. Bachilo, Nano Lett. 3, 1235 (2003).

${ }^{3}$ R. B. Capaz, C. D. Spataru, S. Ismail-Beigi, and S. G. Louie, Phys. Rev. B 74, 121401 (2006).

${ }^{4}$ Z. Wang, H. Zhao, and S. Mazumdar, Phys. Rev. B 74, 195406 (2006).

${ }^{5}$ J. Jiang, R. Saito, G. G. Samsonidze, A. Jorio, S. G. Chou, G. Dresselhaus, and M. S. Dresselhaus, Phys. Rev. B 75, 035407 (2007).

${ }^{6}$ K. Sato, R. Saito, J. Jiang, G. Dresselhaus, and M. S. Dresselhaus, Phys. Rev. B 76, 195446 (2007).

${ }^{7}$ T. Ando, J. Phys. Soc. Jpn. 78, 104703 (2009).

${ }^{8}$ T. Ando, J. Phys. Soc. Jpn. 66, 1066 (1997).

${ }^{9}$ T. Ando, J. Phys. Soc. Jpn. 73, 3351 (2004).

${ }^{10}$ M. Ichida, S. Mizuno, Y. Tani, Y. Saito, and A. Nakamura, J. Phys. Soc. Jpn. 68, 3131 (1999).

${ }^{11}$ M. Ichida, S. Mizuno, Y. Saito, H. Kataura, Y. Achiba, and A. Nakamura, Phys. Rev. B 65, 241407 (2002).
${ }^{12}$ M. J. O’Connell et al., Science 297, 593 (2002).

${ }^{13}$ F. Wang, G. Dukovic, L. E. Brus, and T. F. Heinz, Science 308, 838 (2005).

${ }^{14}$ J. Maultzsch, R. Pomraenke, S. Reich, E. Chang, D. Prezzi, A. Ruini, E. Molinari, M. S. Strano, C. Thomsen, and C. Lienau, Phys. Rev. B 72, 241402 (2005).

${ }^{15}$ H. Zhao, S. Mazumdar, C.-X. Sheng, M. Tong, and Z. V. Vardeny, Phys. Rev. B 73, 075403 (2006).

${ }^{16}$ C. D. Spataru, S. Ismail-Beigi, L. X. Benedict, and S. G. Louie, Phys. Rev. Lett. 92, 077402 (2004).

${ }^{17}$ C. D. Spataru, S. Ismail-Beigi, R. B. Capaz, and S. G. Louie, Phys. Rev. Lett. 95, 247402 (2005).

${ }^{18}$ E. Chang, G. Bussi, A. Ruini, and E. Molinari, Phys. Rev. Lett. 92, 196401 (2004).

${ }^{19}$ E. Chang, G. Bussi, A. Ruini, and E. Molinari, Phys. Rev. B 72, 195423 (2005)

${ }^{20}$ V. Perebeinos, J. Tersoff, and Ph. Avouris, Phys. Rev. Lett. 92, 257402 (2004)

${ }^{21}$ H. Zhao and S. Mazumdar, Phys. Rev. Lett. 93, 157402 (2004). 
${ }^{22}$ C. L. Kane and E. J. Mele, Phys. Rev. Lett. 93, 197402 (2004).

${ }^{23}$ H. Ajiki and T. Ando, Phys. B (Amsterdam) 201, 349 (1994).

${ }^{24}$ H. Ajiki and T. Ando, Jpn. J. Appl. Phys. Suppl. 34-1, 107 (1995).

${ }^{25}$ S. Uryu and T. Ando, Phys. Rev. B 74, 155411 (2006).

${ }^{26}$ Z.-D. Wang, H.-B. Zhao, and S. Mazumdar, Phys. Rev. B 76, 115431 (2007).

${ }^{27}$ Y. Miyauchi, M. Oba, and S. Maruyama, Phys. Rev. B 74, 205440 (2006).

${ }^{28}$ J. Lefebvre and P. Finnie, Phys. Rev. Lett. 98, 167406 (2007).

${ }^{29}$ K.-C. Chuang, A. Nish, J.-Y. Hwang, G. W. Evans, and R. J. Nicholas, Phys. Rev. B 78, 085411 (2008).

${ }^{30}$ T. Ando, J. Phys. Soc. Jpn. 74, 777 (2005).

${ }^{31}$ T. Ando, J. Phys. Soc. Jpn. 79, 024706 (2010).

${ }^{32}$ C. L. Kane and E. J. Mele, Phys. Rev. Lett. 78, 1932 (1997).

${ }^{33}$ Vl. A. Margulis and T. A. Sizikova, Phys. B (Amsterdam) 245, 173 (1998).

${ }^{34}$ D. S. Novikov and L. S. Levitov, Phys. Rev. Lett. 96, 036402 (2006).

${ }^{35}$ H. Ajiki and T. Ando, J. Phys. Soc. Jpn. 65, 505 (1996).

${ }^{36}$ T. Ando, J. Phys. Soc. Jpn. 69, 1757 (2000).

${ }^{37}$ C. L. Kane et al., Europhys. Lett. 41, 683 (1998).

${ }^{38}$ H. Suzuura and T. Ando, Phys. E (Amsterdam) 6, 864 (2000).

${ }^{39}$ H. Suzuura and T. Ando, Mol. Cryst. Liq. Cryst. 340, 731 (2000).

${ }^{40}$ H. Suzuura and T. Ando, Phys. Rev. B 65, 235412 (2002).

${ }^{41}$ H. Ajiki, J. Phys.: Conf. Ser. 210, 012049 (2010).

${ }^{42}$ S. Uryu and T. Ando, Phys. Rev. B 76, 115420 (2007).

${ }^{43}$ Y. Miyauchi, H. Ajiki, and S. Maruyama, Phys. Rev. B 81, 121415 (2010).

${ }^{44}$ H. Ajiki and T. Ando, J. Phys. Soc. Jpn. 62, 1255 (1993).

${ }^{45}$ J. C. Slonczewski and P. R. Weiss, Phys. Rev. 109, 272 (1958).

${ }^{46}$ H. Suzuura and T. Ando, Phys. Rev. Lett. 89, 266603 (2002).

${ }^{47}$ T. Ando, J. Phys. Soc. Jpn. 75, 054701 (2006).

${ }^{48}$ S. Uryu, H. Ajiki, and T. Ando, Phys. Rev. B 78, 115414 (2008).

${ }^{49}$ R. Saito, G. Dresselhaus, and M. S. Dresselhaus, Physical Properties of Carbon Nanotubes (Imperial College Press, London, 1998).

${ }^{50}$ T. Ando, J. Phys. Soc. Jpn. 75, 024707 (2006).

${ }^{51}$ H. Sakai, H. Suzuura, and T. Ando, J. Phys. Soc. Jpn. 72, 1698 (2003).

${ }^{52}$ H. Sakai, H. Suzuura, and T. Ando, Phys. E (Amsterdam) 22, 704 (2004).

${ }^{53}$ V. Perebeinos, J. Tersoff, and Ph. Avouris, Nano Lett. 5, 2495 (2005).

${ }^{54}$ S. Zaric et al., Phys. E (Amsterdam) 29, 469 (2005).

${ }^{55}$ I. B. Mortimer and R. J. Nicholas, Phys. Rev. Lett. 98, 027404 (2007).
${ }^{56}$ I. B. Mortimer, L.-J. Li, R. A. Taylor, G. L. J. A. Rikken, O. Portugall, and R. J. Nicholas, Phys. Rev. B 76, 085404 (2007).

${ }^{57}$ J. Shaver, J. Kono, O. Portugall, V. Krsti, G. L. J. A. Rikken, Y. Miyauchi, S. Maruyama, and V. Perebeinos, Nano Lett. 7, 1851 (2007).

${ }^{58}$ J. Shaver and J. Kono, Laser Photon. Rev. 1, 260 (2007).

${ }^{59}$ A. Srivastava, H. Htoon, V. I. Klimov, and J. Kono, Phys. Rev. Lett. 101, 087402 (2008).

${ }^{60}$ J. Shaver, S. A. Crooker, J. A. Fagan, E. K. Hobbie, N. Ubrig, O. Portugall, V. Perebeinos, Ph. Avouris, and J. Kono, Phys. Rev. B 78, 081402 (2008).

${ }^{61}$ R. Matsunaga, K. Matsuda, and Y. Kanemitsu, Phys. Rev. Lett. 101, 147404 (2008)

${ }^{62}$ R. Matsunaga, Y. Miyauchi, K. Matsuda, and Y. Kanemitsu, Phys. Rev. B 80, 115436 (2009).

${ }^{63}$ R. Kubo, J. Phys. Soc. Jpn. 12, 570 (1957).

${ }^{64}$ W. P. Chen, Y. J. Chen, and E. Burstein, Surf. Sci. 58, 263 (1976).

${ }^{65}$ S. J. Allen Jr., D. C. Tsui, and B. Vinter, Solid State Commun. 20, 425 (1976).

${ }^{66}$ T. Ando, Z. Phys. B: Condens. Matter Quanta 26, 263 (1977).

${ }^{67}$ S. G. Chou et al., Chem. Phys. Lett. 397, 296 (2004).

${ }^{68}$ C. Fantini, A. Jorio, M. Souza, M. S. Strano, M. S. Dresselhaus, and M. A. Pimenta, Phys. Rev. Lett. 93, 147406 (2004).

${ }^{69}$ T. Hertel, A. Hagen, V. Talalaev, K. Arnold, F. Hennrich, M. Kappes, S. Rosenthal, J. McBride, H. Ulbricht, and E. Flahaut, Nano Lett. 5, 511 (2005).

${ }^{70}$ D. A. Tsyboulski, S. M. Bachilo, and R. B. Weisman, Nano Lett. 5, 975 (2005).

${ }^{71}$ Y. Ohno, S. Iwasaki, Y. Murakami, S. Kishimoto, S. Maruyama, and T. Mizutani, Phys. Rev. B 73, 235427 (2006).

${ }^{72}$ Y. Miyauchi, R. Saito, K. Sato, Y. Ohno, S. Iwasaki, T. Mizutani, J. Jiang, and S. Maruyama, Chem. Phys. Lett. 442, 394 (2007).

${ }^{73}$ P. T. Araujo and A. Jorio, Phys. Status Solidi B 245, 2201 (2008).

${ }^{74}$ A. Stahl and I. Balslev, Electrodynamics of Semiconductor Band Edge, Springer Tracts in Modern Physics, Vol. 110 (Springer Verlag, Berlin, 1987).

${ }^{75}$ K. Cho, Suppl. Prog. Theor. Phys. 106, 225 (1991).

${ }^{76}$ O. Keller, Phys. Rep. 268, 85 (1996).

${ }^{77}$ H. Ajiki, T. Iida, T. Ishikawa, S. Uryu, and H. Ishihara, Phys. Rev. B 80, 115437 (2009).

${ }^{78}$ M. Rohlfing and S. G. Louie, Phys. Rev. Lett. 80, 3320 (1998).

${ }^{79}$ M. Rohlfing and S. G. Louie, Phys. Rev. B 62, 4927 (2000).

${ }^{80}$ A. Fetter and J. D. Walecka, Quantum Theory of Many Particle Systems (Dover, New York, 2003). 